\title{
Immunoglobulin heavy chains in medaka (Oryzias latipes)
}

Susana Magadán-Mompó ${ }^{1}$, Christian Sánchez-Espinel ${ }^{2}$ and Francisco Gambón-Deza ${ }^{3^{*}}$

\begin{abstract}
Background: Bony fish present an immunological system, which evolved independently from those of animals that migrated to land 400 million years ago. The publication of whole genome sequences and the availability of several cDNA libraries for medaka (Oryzias latipes) permitted us to perform a thorough analysis of immunoglobulin heavy chains present in this teleost.

Results: We identified IgM and IgD coding ESTs, mainly in spleen, kidney and gills using published cDNA libraries but we did not find any sequence that coded for IgT or other heavy chain isotypes described in fish. The IgM ESTs corresponded with the secreted and membrane forms and surprisingly, the latter form only presented two constant heavy chain domains. This is the first time that this short form of membrane lgM is described in a teleost. It is different from that identified in Notothenioid teleost because it does not present the typical splicing pattern of membrane IgM. The identified IgD-ESTs only present membrane transcripts, with $C \mu 1$ and five $C \delta$ exons.

Furthermore, there are ESTs with sequences that do not have any VH which disrupt open reading frames.

A scan of the medaka genome using transcripts and genomic short reads resulted in five zones within a region on chromosome 8 with $\mathrm{C} \mu$ and $\mathrm{C} \delta$ exons. Some of these exons do not form part of antibodies and were at times interspersed, suggesting a recombination process between zones. An analysis of the ESTs confirmed that no antibodies are expressed from zone 3 .
\end{abstract}

Conclusions: Our results suggest that the IGH locus duplication is very common among teleosts, wherein the existence of a recombination process explains the sequence homology between them.

\section{Background}

Genome information of vertebrates is rapidly becoming available thanks to several full vertebrate genome projects. Such information is very useful for comparative and evolutionary biologists. Comparative genomic studies are helping to discover evolutionary mechanisms that underlie diversification of organisms $[1,2]$. Therefore, information obtained from genomes is of great use for understanding the genetic basis of antibody diversity and the evolutionary divergences of the immunoglobulin locus in vertebrates [3]. Immunoglobulin loci are organised into two main types called: "cluster" and "translocon". Cluster type organization is found in both light and heavy chain loci of cartilaginous fish $[4,5]$ There are many independent variable $(\mathrm{VH})$, diversity $(\mathrm{D})$, joining

\footnotetext{
* Correspondence: fgambon@gmail.com

${ }^{3}$ Unidad de Inmunología, Hospital do Meixoeiro, Servizo Galego de Saude

(SERGAS), Carretera de Madrid s/n, Vigo 36210, Pontevedra, Spain

Full list of author information is available at the end of the article
}

$(\mathrm{JH})$ and constant $(\mathrm{CH})$ segments sets $[\mathrm{VH}(\mathrm{D}) \mathrm{JHCH}]$ along wide areas of the genome. Therefore, diversity in these molecules is generated through synthesis of antibodies from each of these VH-D-JH-CH regions [6,7]. In tetrapods and bony fish, the IGH locus configuration is translocon and it presents some specific characteristics. There are genomic segments for the variable regions of antibody heavy chains $(\mathrm{VH})$ and these are followed by segments that code for: diversity (D), joining $(\mathrm{JH})$, and segments that encode the heavy chain domains $(\mathrm{CH})$. A rearranged VHDJH region spliced to $\mathrm{CH}$ segment is needed to generate an antibody $[8,9]$.

It is well established that all fishes have IGHM and other constant chain region genes in the 3' region. Dooley and Flajnik described genes that encoded the IgW (omega immunoglobulin isotype) and IgNAR (New Antigen Receptor) antibodies in the 3' region, for cartilaginous fish [10-12]. Most bony fish belong to the infraclass teleost, where we can find IgM, IgD [13-15] 
and IgT/IgZ [16]. However, the IgT/IgZ have not been found in catfish [14]. Teleost IgD is an antibody which generally has seven domains and some of these have experienced recent duplications [17]. The IGHZ (of zebrafish) and IGHT (of rainbow trout) correspond to genes that code for antibodies (IgZ and IgT) with four immunoglobulin domains located upstream from the D and $\mathrm{JH}$ segments of IGHM. Furthermore, the exons that code for the constant region present their own D and $\mathrm{JH}$ segments, and resemble the organization of $\mathrm{T}$ cell receptor alpha and delta (TCR $\alpha$ and $\delta$ ) loci [18]. Other genes for antibodies found at the same location were described later, and may correspond to different forms of the same antibody $[17,19]$.

Another surprising feature found in some teleost IGH loci, such as in stickleback, catfish $[14,17]$ and medaka, is the presence of core block $[\mathrm{VH}(\mathrm{D}) \mathrm{JHCH}]$ duplications in the germline. Such presence is perhaps not widespread in teleosts because they were not found in zebrafish genome [16]. The duplications present a high homology suggesting that they happened recently or perhaps there is a biological mechanism that maintains them.

This article presents a description of the antibodies in medaka, wherein antibody structure was deduced based on genomic and EST data. Five zones or regions that code for constant chain immunoglobulin domains have been found in genome, and each of these regions has exons for IgM and IgD. Medaka (Oryzias latipes), catfish (Ictalurus punctatus), zebrafish (Danio rerio) and stickleback (Gasterosteus aculeatus) represent a group of teleosts that have been widely used as animal models in various fields such as biology, medicine, environmental science and fisheries [20,21]. There is ample information on zebrafish, catfish and stickleback immunoglobulin loci but this is the first time that work on medaka immunoglobulins is published.

\section{Methods}

\section{Fish and sampling}

Adult medaka (Oryzias latipes, strain HdrR belongs to the Southern Japanese population) specimens were kindly supplied by J. Cerdá (Institute of Marine Sciences of Barcelona, CSIC, and Aquaculture Centre). Fish were killed by overexposure to MS222 (Sigma Chemicals). Head kidney and spleen were removed aseptically and RNA was extracted immediately using the QIAmp RNA kit (QIAGEN) following manufacturer's instructions.

\section{CDNA preparation, PCR and DNA sequencing}

About $5 \mu \mathrm{g}$ of total RNA was reverse transcripted into cDNA by using QIAGEN One Step RT-PCR kit and priming with $0.5 \mu \mathrm{M}$ of C 86 -antisense primer (5'GGACTGTTGGAGGATTCATGTCTCACA-3') in a total volume of $50 \mu$ l.
Amplification of the IgD constant region was performed in a two-step PCR reaction. $5 \mu \mathrm{l}$ of cDNA reaction mixture was amplified by thermal cycling in a total volume of $25 \mu \mathrm{l}$ using $\mathrm{C} \mu 1$-sense (5'-CATTGACTTT CTCATGGACTCAGGGC-3') combined with C86- antisense primer. Amplification was performed for 30 cycles at $95^{\circ} \mathrm{C} 30 \mathrm{~s}, 65^{\circ} \mathrm{C}$ for $30 \mathrm{~s}$ and $72^{\circ} \mathrm{C}$ for $90 \mathrm{~s}$, with a final elongation step at $72^{\circ} \mathrm{C}$ for $10 \mathrm{~min}$. Due to a very low amplification product obtained from the first PCR, a second round was performed for 20 more cycles using the same primers and conditions. The amplified products were sequenced on an Applied Biosystems 3130 Genetic Analyzer. The Gepard (GEnome PAir - Rapid Dotter) program [22] was used to search for homologues with the genomic sequences and identify the IgD domains.

\section{Medaka immunoglobulin expression using ESTs databases}

Previously identified immunoglobulin constant heavy chain exons from stickleback [17] were used to search homologue sequences in the medaka ESTs database (http://www.shigen.nig.ac.jp/medaka). A total of 11 cDNA libraries generated from different tissues of HdrRmedaka were scanned (Additional file 1). ESTs encoding for IgM and/or IgD were retrieved. The medaka immunoglobulin ESTs can be found grouped into three clusters: a) CLSTF16513, with the 5' sequences encoding IgM and IgD, b) CLSTR12908 with 3' sequences for IgM and c) CLSTR18886 with 3' IgD sequences.

In order to identify the genomic zone or region that corresponds to each EST, an alignment was performed using the Lastz program available at the Galaxy website (http://main.g2.bx.psu.edu/) [23,24]. To confirm results we performed the same analysis using recently released next generation RNA sequences (SRA023697) deposited in the Sequence Read Archive database of the NCBI (http://www.ncbi.nlm.nih.gov/sra). These alignments were visualized using the Tablet - Next Generation Sequence Assembly Visualization software (http://bioinf. scri.ac.uk/tablet/) [25].

\section{Identification of the IGH locus}

The complete genome of Oryzias latipes (assembly: HdrR, October 2005; version 56.1i) built in NCBI (http:// www.ncbi.nlm.nih.gov) and Ensembl database platforms (http://www.ensembl.org/index.html) was examined to locate antibody genes. Previously published sequences from other IGHM teleost fish were used to identify genomic scaffolds and chromosomes that contained immunoglobulin genes. These sequences (scaffolds 146, 409 and 501 , chromosome 8 ) were retrieved and analysed in detail using the Vector-NTI (Invitrogen). Two scaffolds were not assigned to any chromosome (scaffold 3172 and 
1447) but were identified as harboring IGH gene segments and these scaffolds were observed to overlap on 400 nucleotides suggesting that they are contiguous (Additional File 2).

Identification of exons coding for $\mathrm{CH}$ domains was performed by aligning genomic sequences with previously published immunoglobulin mRNAs. Limits of unpublished antibodies were deduced following instructions in the software FGNESH (http://www.softberry. com) and Augustus (http://augustus.gobics.de/submission) [26]. Messenger RNA predicted from the gene sequence was compared with $\mathrm{O}$. latipes EST sequences from NCBI and http://www.shigen.nig.ac.jp/medaka, in order to confirm exon ends and analyse gene expression.

The heavy chain variable segments $(\mathrm{VH})$ of medaka were located on the same scaffolds and chromosome. Several criteria were used to identify VH segments, including: a) the presence of recombination signal sequences (RSS) including the canonical "tattattgt" nonamer sequences (allowing 1 or 2 nucleotide mismatches) and corresponding heptamer sequences, b) the presence of AG and and GT splice sides flanking open reading frames, and c) pattern searches for identifying RSS with 23 bp spacers flanking the 3'end of the $\mathrm{VH}$ regions. We verified whether the read sequences corresponded to the $\mathrm{VH}$ regions [27].

D segments were identified by the presence of RSS 5' and RSS 3' [24]. They were compared with O. latipes EST database in order to confirm their expression. The heavy chain joining $(\mathrm{JH})$ segments were located by homology to published $\mathrm{JH}$ sequences. This was carried out by comparing a dot plot between published $\mathrm{JH}$ sequences and the 5 ' region of the IGHM (implementing a window of $30 \mathrm{nt}$ and a match of $60 \%$ ). RSS was used to detect the beginning of the $\mathrm{JH}$ exon while the presence of "GTA" was used to determine the end [27].

The immunoglobulin gene nomenclature used to describe the identified genes followed the guidelines of the international ImMunoGeneTics Information System (http://imgt.org) [28].

In order to resolve occasional mistakes and complete the gaps, all scaffolds retrieved (scaffolds 146, 409 and 501, chromosome 8) were aligned with the recently released genomic new generation sequences (DRA000220), deposited in the Sequence Read Archive database of the NCBI (http://www.ncbi.nlm.nih.gov/sra). The in silico analysis was carried out using the available tools at the Galaxy website (http://main.g2.bx.psu.edu/) and visualized with Tablet - Next Generation Sequence Assembly Visualization software (http://bioinf.scri.ac.uk/tablet/).

\section{Phylogenetic studies}

Comparative phylogenetic studies were carried out with the program MEGA5 [29] using the algorithm to perform BLOSSUM alignments. The neighbour-joining and minimum evolution methods were then used to plot the phylogenetic trees (pair-wise deletion, JonesTaylor-Thornton matrix and enter range activated sites (gamma-number 2.5). The veracity of these trees was studied using the above-mentioned method and by executing 1000 replicate bootstrappings.

\section{GenBank sequences}

IgM accession numbers: X83372 Oncorhynchus mykiss (rainbow trout), AB2 17624 Takifugu rubripes (Fugu rubripes), AAQ14862 Sineperca chuatsi, AAF69488 Hippoglossus hippoglossus, A46538 Gadus morhua (Atlantic cod), AAO37747 Ornithorhynchus anatinus (platypus) and EU287910 and EU28791 1 Eublepharis macularius (leopard gecko). The G. aculeatus IGH sequences are in the supplementary information (Additional File 3) [17].

\section{Results}

\section{Immunoglobulins in medaka}

A bioinformatic search of ESTs in the NBRP medaka database (http://www.shigen.nig.ac.jp/medaka/) was carried out in order to determine the kind of antibodies expressed by the teleost medaka. Previously published $\mathrm{C} \mu, \mathrm{C} \delta, \mathrm{C} \zeta$, sequences from $G$. aculeatus [17] were used as queries to identify the ESTs.

A total of 94 EST sequences of IgM and 19 of IgD were identified but we did not find any EST homologues to IgT/Z. Only ESTs with data from both ends (considered full length) were analysed further and are summarized according to their tissue distribution (Table 1). As already reported in other teleosts, IgM and IgD genes are mainly expressed in kidney, gills and spleen. And only a few IgM ESTs were detected in liver, ovarian tissue and brain.

As shown below, approximately $15 \%$ of these ESTs present atypical rearrangement and stop codons in all reading frames. Three immunoglobulin heavy chain forms were found among the viable ESTs. Two of these corresponded to the IgM: transmembrane and secreted forms, and one to transmembrane IgD (Figure 1). Surprisingly, the IgM transmembrane form presented only two $\mathrm{CH}$ domains $(\mathrm{C} \mu 1$ and $\mathrm{C} \mu 2)$, TM1 and TM2. The deduced amino acid sequence of medaka $\operatorname{IgM}$ showed a cysteine residue in the $\mathrm{CH} 1$ domain which is involved in the formation of disulphide bonds with the light chain. We did not find any cysteine in $\mathrm{CH} 1$ and $\mathrm{CH} 2$ to

Table 1 Tissue distribution for immunoglobulin ESTs

\begin{tabular}{ccccccc}
\hline & Gills & Kidney & Spleen & Liver & Ovarian & Brain \\
\hline IgM - EST & 10 & 45 & 35 & 1 & 2 & 1 \\
IgD - EST & $1(1)^{*}$ & $11(3)^{*}$ & $7(5)^{*}$ & 0 & 0 & 0 \\
\hline
\end{tabular}

* The number in brackets represents the IgD sequences with $C \mu 1$. 


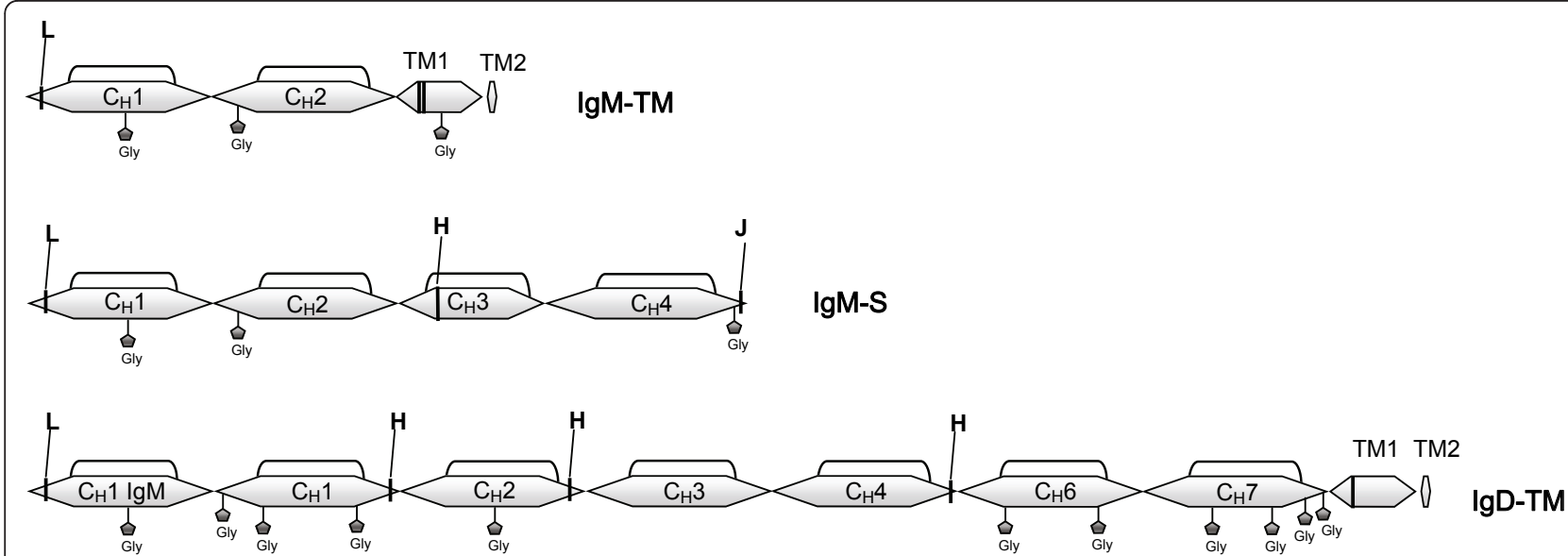

Figure 1 Immunoglobulin heavy chain constant domains. The protein structure was deduced from the mRNA coding for transmembrane $\lg M(\operatorname{lgM}-T M)$, secreted $\lg M(\operatorname{lgM}-S)$ and transmembrane $\lg D$ (IgD-TM) forms. The putative cysteines that establish bonds with light chains (L) and heavy chains $(H)$ to form multimers $(J)$ are shown. Potential glycosylation sites are indicated with Gly.

join the heavy chains; however there were two cysteines in TM1 that may be responsible for covalent heavy chain binding.

The secreted IgM form appears to be similar to those described in other teleostei [14], with four $\mathrm{CH}$ domains and a secretory tail. This IgM presents three cysteines for interchain bonds, one in the $\mathrm{CH} 1$ domain to establish a disulfide bond with the light chain, another in the $\mathrm{CH} 3$ domain to join heavy chains and finally, in the secretory tail, probably to form multimers (Additional File 4).

The study of IgD ESTs permitted us to deduce its structure. This is similar to those described in other teleostei, in which the first constant domain is $C \mu 1$ followed by $\mathrm{C} \delta 1$. The $\mathrm{C} \delta 6, \mathrm{C} \delta 7$, TM1 and TM2 domains were present in the ESTs in all cases. All IgD domains expressed could not be described because the forward and reverse ESTs sequences did not overlap. Thus, we decided to perform a RT-PCR of head kidney and spleen mRNA with primers designed for the $\mathrm{C} \mu 1$ and C $\delta 6$. A PCR product of approximately $1600 \mathrm{bp}$ was obtained and its sequencing confirmed the presence of $\mathrm{C} \delta 1, \mathrm{C} \delta 2$, $\mathrm{C} \delta 3, \mathrm{C} \delta 4$ and $\mathrm{C} \delta 6$ domains (Additional File 5). There was no $\mathrm{C} \delta 5$ equivalent in all IgD transcripts sequenced.

\section{Medaka IGH Genomic organization}

Four different sequences of IgM in ESTs were found, suggesting several isotypes or allotypes. These were used to scan the medaka genome and map immunoglobulin heavy chain genes. Several sequences were found on chromosome eight (scaffolds 146, 409 and 501) that cover approximately $450 \mathrm{~kb}$ (Figure 2). We also identified two scaffolds that were not assigned to any chromosome (scaffolds 3172 and 1447, with 400 nucleotides overlapped suggesting that they are contiguous) in which several immunoglobulin heavy chain coding exons were annotated.

We were able to elucidate the IGH genomic organization, despite finding several gaps, mainly between scaffolds and contigs, which prevent us from creating a complete contiguous annotation. Furthermore, we were able to complete some gaps and solve several contradictions found between ESTs and genomic sequences, using recently deposited next generation sequence data (DRA000220 and SRA026397) in the Sequence Read Archive Database (http://www.ncbi.nlm.nih.gov/sra).

In order to identify the immunoglobulin heavy chain genes, the genomic scaffolds were divided into $30 \mathrm{~Kb}$ segments and analysed using the FGNESH and Augustus software packages [23]. The obtained results were verified through a dot plot and Est2genome (EMBOSS) with available medaka ESTs that had been identified as immunoglobulins. As shown in Figure 2 and 3, the IGH genomic organization is complex, with five specific zones where $\mathrm{C} \mu$ and $\mathrm{C} \delta$ exons are identified (In Additional File 6 you can find the nucleotide sequences of all zones in GenBank format, we have included a file with $\mathrm{VH}$ annotation). It is worthy to note that not all exons are expected to produce an immunoglobulin heavy chain in most zones. Therefore, in order to avoid confusion, instead of naming exons according to their numerical order of appearance in the genomic sequence, we decided to number them according to their orthologous exons defined in other species. The Additional file 7 shows a tree constructed using the aligned medaka $\mathrm{CH}$ amino acid sequences and $\mathrm{CHs}$ deduced from sequences of the bony fish stickleback (G. aculeatus) which supports this criterion for classification.

Overall, $\mathrm{C} \mu$ and $\mathrm{C} \delta$ exons, D and $\mathrm{JH}$ segments (Figure 3) were identified in each of the five genomic zones. No 


\section{Locus IGH - Chromosome 8}

\section{$452681 \mathrm{bp}$}

\section{Scaffold 409}

Scaffold 501

Scaffold 146
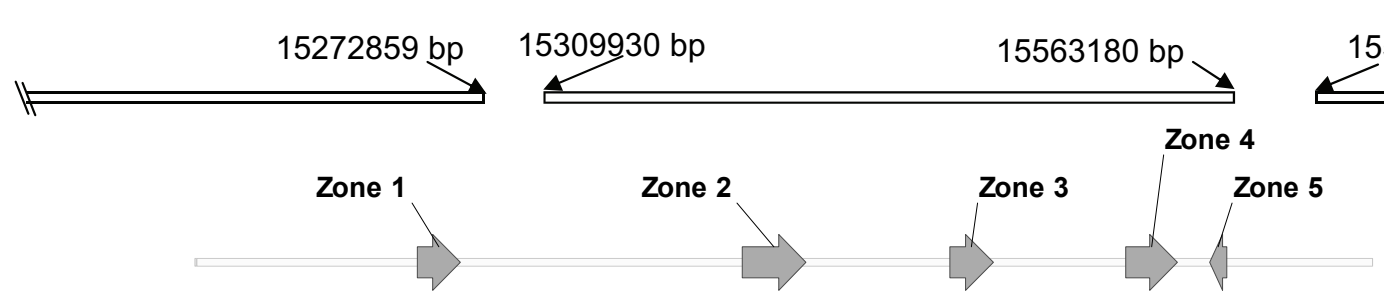

\section{Contigs}

$15166586 \mathrm{bp}$

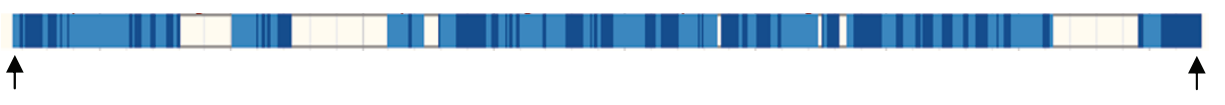

15619266 bp

Figure 2 The IGH locus of $\boldsymbol{O}$. latipes. The image displays the location of scaffolds that encode immunoglobulin heavy chain genes in chromosome 8. Five specific zones can be deduced in which $\mathrm{C} \mu$ and $C \delta$ exons are identified. Contigs that cover the IGH locus are represented at the bottom in dark and light blue. The white segments show gaps in the genomic sequence.

C $\delta$ exons were found and this is consistent with data obtained from ESTs analysis. As indicated in Figure 3 some exons were identified or corrected based on the analysis of ESTs and the next generation sequence data (SRA026397 and DRA000220). VH regions were found between zones (see Additional File 6 and 8).

Another important feature is the sequence homology between different zones as shown in Figure 4. A dot plot of zone 1 versus zone 4 genomic sequences elucidated duplications of large and small segments, suggesting a recombination process. The similarity between the sequences of homologous domains and the flanking introns indicates that this process must have taken place recently.

The genomic region designated as zone 1 encodes seven $\mathrm{JH}$ segments followed by $\mathrm{C} \mu 1, \mathrm{C} \mu 2, \mathrm{C} \mu 3$ and $\mathrm{C} \mu 4$, and harbors exons that code for a transmembrane and cytoplasm domain. In this zone there are only four $\mathrm{C} \delta$ exons ( $\mathrm{C} \delta 1, \mathrm{C} \delta 2, \mathrm{C} \delta 6$ and $\mathrm{C} \delta 7$ ) located $3 \mathrm{~kb}$ downstream of the nearest $\mathrm{C} \mu$ and are followed by transmembrane and cytoplasm exons. There is a gap between $\mathrm{C} \delta 2$ and $\mathrm{C} \delta 6$, where there is a high probability of finding the presence of $\mathrm{C} \delta$ exons in this first zone.

The remaining zones give us an idea of asymmetric duplications, that is, the presence of $\mathrm{C} \mu$ and $\mathrm{C} \delta$ exons with a changed configuration. In zone 2 , just like in zone 1, the exons $C \mu 1, C \mu 2, C \mu 3$ (also deduced from EST sequences) and $C \mu 4$ appear after seven D segments and seven $\mathrm{JH}$ segments (Figure 3). Exons for transmembrane and cytoplasm domains are also present. At 3' of these exons, we find one $C \delta 2$ exon without any other sequence coding for IgD antibody. Interestingly, about $5 \mathrm{~kb}$ downstream, we find $\mathrm{D}$ and $\mathrm{JH}$ segments followed by $\mathrm{C} \mu 1$ and C81- C82- C83- C84- C86 exons again. Therefore, we can differentiate two genomic regions in this zone, namely; zone $2 \mathrm{a}$ at 5', and zone $2 \mathrm{~b}$ at 3 '. Both of them have exons to IgM and IgD.

The zone designated as zone 3 seems to be quite disorganized when compared with other zones. As shown in Figure 3, there are very few exons and this suggests that this zone may not generate functional antibodies. Conversely, zone 4 appears to be well structured and presents the highest number of exons. At the 5' region there are four $\mathrm{C} \mu$ exons, including $\mathrm{C} \mu 2$ with their transmembrane and cytoplasm coding exons (Figure 3). Surprisingly, domain $\mathrm{C} \mu 4$ and the transmembrane and cytoplasm exons are found to be duplicated. At the 3' region, there are 10 exons for IgD domains, some of which are repeated $(\mathrm{C} \delta 2, \mathrm{C} \delta 3, \mathrm{C} \delta 4$ and $\mathrm{C} \delta 6)$. Between the last $\mathrm{C} \delta 6$ and the $\mathrm{C} \delta 7$ there are exons that code for $\operatorname{IgM}(\mathrm{C} \mu 2$ and $\mathrm{C} \mu 4)$ and finally, we find sequences for transmembrane and cytoplasm IgD domains.

At present, zone 5 is the least resolved genomic region. This is due to the presence of a gap of about 30 $\mathrm{kb}$ between scaffold 501 and the 146 junction. The identified sequences, D segments and C $\delta$ exons, are found to be inverted. The IGHM might be missing due to the presence of the gap however; it is very probable that 


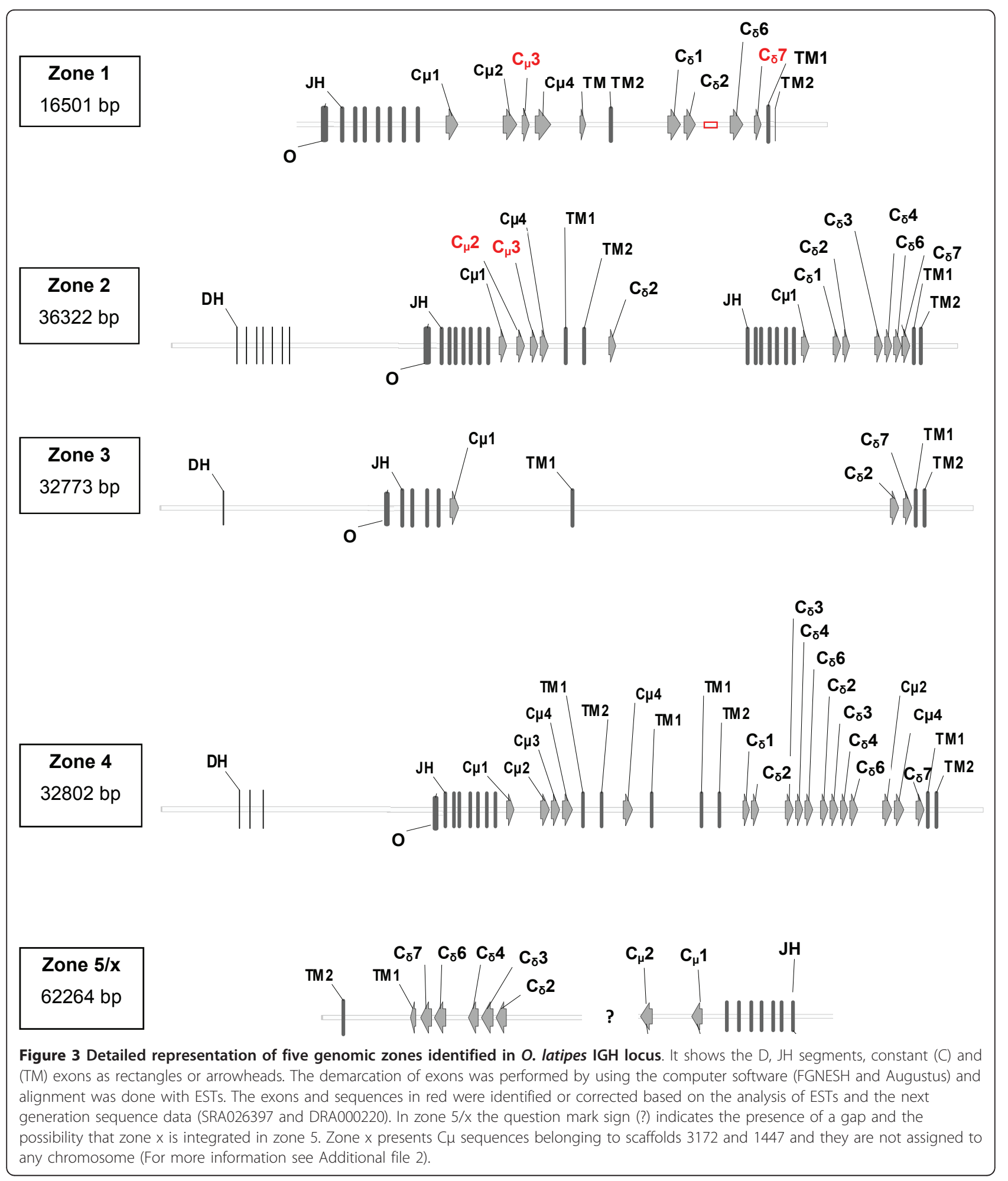

scaffolds 3172 and 1447 belong to the gap because they are not assigned to any chromosome and present sequences for IgM domains (See Figure 3). The above will be taken into account and from now on we will be referred to as zone $5 / \mathrm{x}$.

\section{Correlation between ESTs and genomic sequences}

The structure of this multiple locus with a high number of repetitions and with exons situated out of normal locations made us verify this annotation. To do this we aligned the ESTs and DNA short reads (DRA000220) 


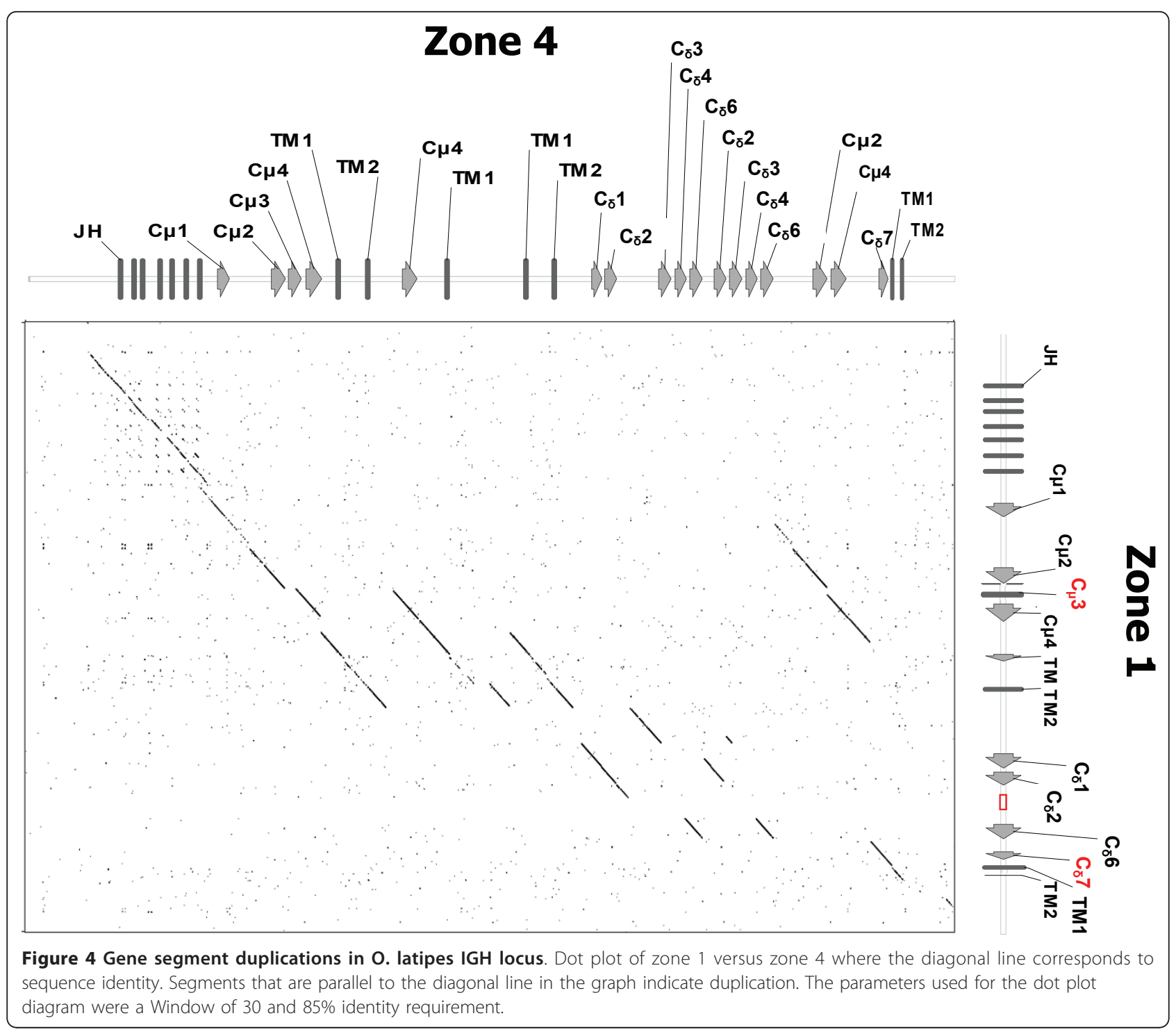

with the different zones of the immunoglobulin locus. For example, Figure 5 shows the alignment of the ESTs with the exon $\mathrm{C} \mu 1$ belongs to zone 2 . We identified ESTs with specific differences that correspond to each of the $C \mu 1$ sequences found in the different zones, except in zone 3 revealing that it is possible to assign ESTs to concordant gene segments (Figure 5). When aligned with the DNA short reads we found the same results but in this case we were able to detect short reads belonging to zone 3 (Additional file 9).

In addition, the tissue distribution of ESTs from lymphoid tissues (kidney, spleen and gills) was correlated with genomic zones. ESTs were found to be expressed from 1, 2, 4 and 5/x zones (Table 2).

On the subject of ESTs coding for IgM, we identified a total of 34 ESTs expressed from zone 1, where 21 corresponded to the secreted form and 13 to the membrane form (Table 2). Thirty-two ESTs were assigned to zone 2, with membrane (6 ESTs) and secreted (26 ESTs) forms. Eleven IgM membrane and five IgM secreted coding ESTs belonged to zone 4. Only 8 IgM ESTs (4 membrane and 4 secreted) were found to be expressed from zone 4 and, as expected due to its disorganized genomic structure, no EST from zone 3 was detected.

It is worthy to note that approximately $15 \%$ of these ESTs (for example the 6 ESTs assigned to zone 4) do not show the classical rearrangement (VH-D-JH-CH) and are expressed without $\mathrm{VH}$ segment. Joining in most of these rearrangements takes place between an exon situated at 3' of $\mathrm{JH}$ segments and $\mathrm{C} \mu 1$. This 3'-JH exon (named Exon 0) has $100 \mathrm{bp}$ and presents stop codons in the 3 ORF (Figure 6). This exon is in zone 1, 2 and 3 and not in 4 and $5 / x$. Not all mRNAs without VH have this exon 0, for example, 6 ESTs assigned to zone 4. 


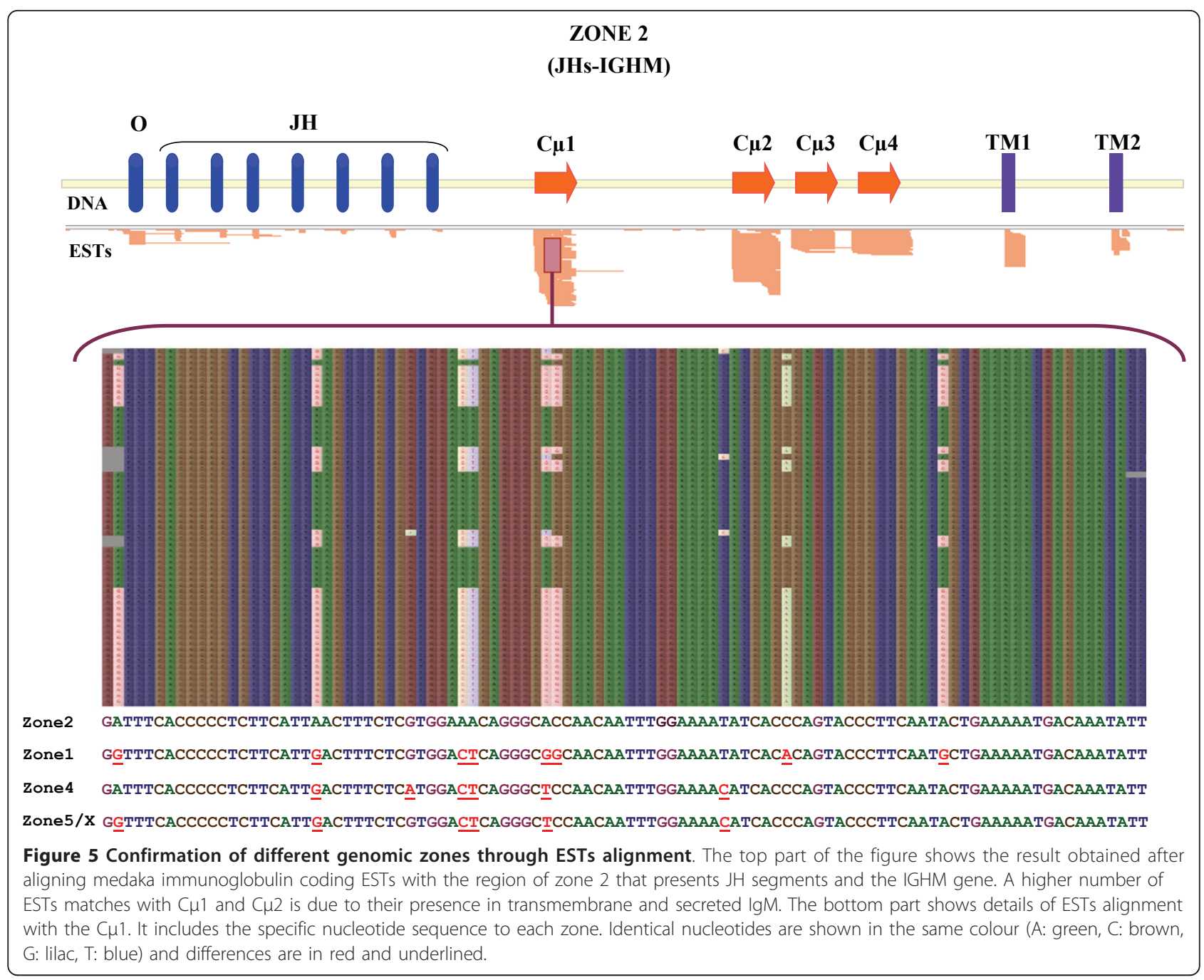

Just like in other fishes, we identified 19 ESTs coding for the membrane IgD form. However, we were unable to unequivocally establish the distribution of these ESTs in each genomic zone because the different $C \delta$ genomic sequences were very similar. Moreover, only four ESTs corresponded to the typical chimeric transcripts observed in teleosts, (utilizing exon $\mathrm{C} \mu 1$ ) and presented the typical VH-D-JH rearrangement. Other IgD transcripts found presented an atypical rearrangement with $\mathrm{C} \delta$ domains and diversity in the 5 ' region, some examples are shown in (Figure 7) where olsp26e01 presents

Table 2 IgM coding ESTs expressed by each genomic zone

\begin{tabular}{cccccc}
\hline & Zone $\mathbf{1}$ & Zone $\mathbf{2}$ & Zone $\mathbf{3}$ & Zone $\mathbf{4}$ & Zone $\mathbf{5 / x}$ \\
\hline IgM-S $^{*}$ & 21 & 26 & 0 & 5 & 2 \\
\hline IgM-TM $^{*}$ & 13 & 6 & 0 & 11 & 2
\end{tabular}

* IgM-S: Secreted Immunoglobulin M.

** IgM-TM: Transmembrane Immunoglobulin M. the exon 0 spliced to $\mathrm{C} \mu 1$, olki34n15 lacks $\mathrm{VH}$ and olsp22p22 includes genomic sequences adjacent to C $\delta 1$.

\section{Discussion}

The analysis of cDNA libraries obtained from different tissues permitted the identification of Ig exons expressed in medaka (Oryzias latipes). ESTs coding for IgM and IgD were identified but no expression or genomic data was found for other isotypes in medaka.

In mammals, the production of secreted and membrane IgM forms involves alternative splicing. The transmembrane form is originated through a cryptic splice site located within $C \mu 4$ that have the acceptor site at 3' of the TM1 exon [30]. This pattern is manifested in Xenopus and cartilaginous fish too [31-33]. However, in teleosts the transmembrane IgM is comprised of the first three exons $(C \mu 1, C \mu 2$ and $C \mu 3)$ plus the transmembrane and cytoplasm exons $[34,35]$. The splicing pattern of IgM appears consistent with exceptions only 


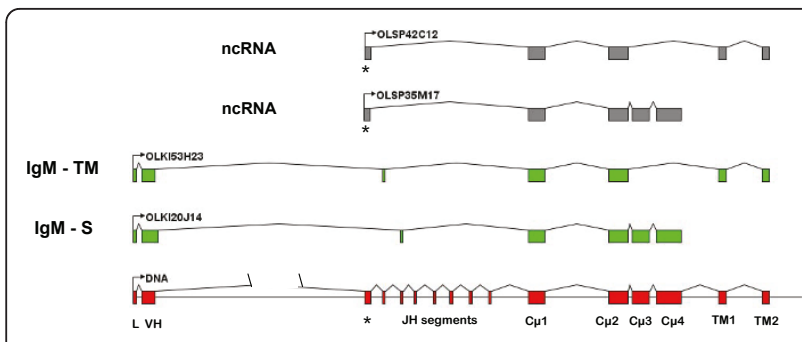

Figure 6 Schematic representation of four IgM ESTs and their relationship with the genomic sequence. Examples of the different RNA splicing found in EST database are indicated. Transcripts for secreted form of IgM (OLKI20J14) with four $\mathrm{C \mu}$ domains and for transmembrane IgM $(\mathrm{OLKI} 53 \mathrm{H} 23)$ with two $\mathrm{C} \mu$ and transmembrane domains are shown. Non coding transcripts (ncRNA) were found too, these included a sequence at the $5^{\prime} \mathrm{JH}$ region named exon $0\left(^{*}\right)$ and have stop codons in the three reading frames.

in a few species, for example, membrane IgM chains with different number of $\mathrm{C} \mu$ domains have been described in ancient fishes [30,36], in which we observe the general rule followed in teleost fishes, $\mathrm{C} \mu 3-\mathrm{TM}$, as well as the mammalian pathway, C $\mu 4$ - TM. However, in Siberian Sturgeon, the splicing pattern can result in a transmembrane immunoglobulin with four, two and half, or only one $\mathrm{C} \mu$ domain [37]. Notothenioid teleosts membrane IgM transcripts likewise lack the $C \mu 3$, and the $C \mu 2$ is spliced to two short exons (RA and $R B$ ) creating an elongated extracellular membrane-proximal domain [38]. Nevertheless, the splicing observed in medaka occurs between the end C $\mu 2$ and TM1 and produces a membrane antigen receptor of only two constant immunoglobulin domains. This is the first time that in a typical teleost is described to have a short

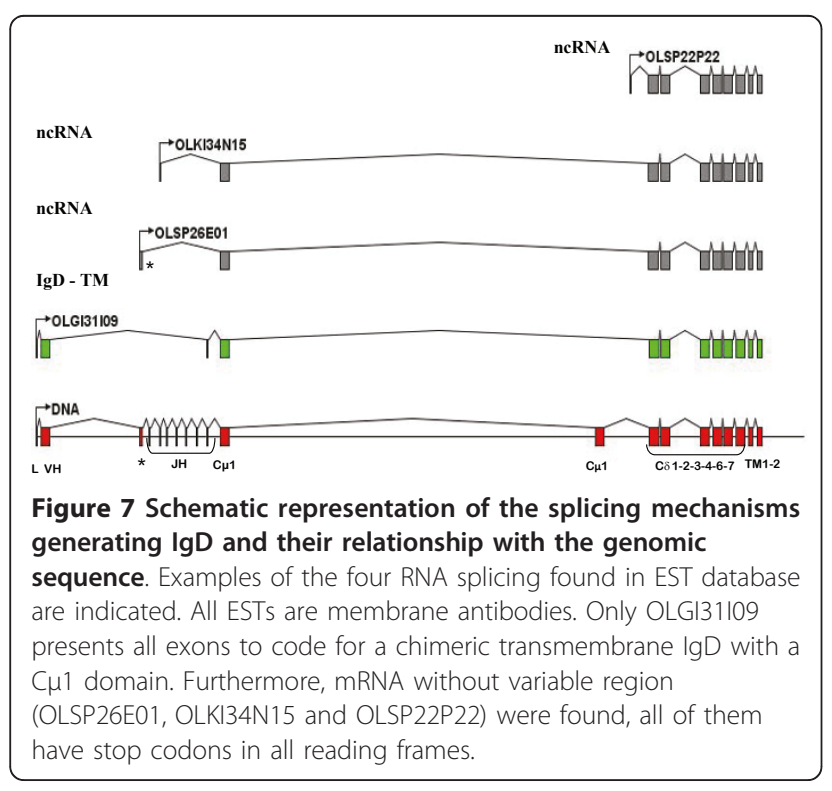

transmembrane IgM and indicates that other teleosts may have evolved to exhibit considerable diversity in IgM splicing. Such diversity may be due to a selection process or due to "genomic configurations" that led to the modification of the splicing machinery.

The medaka IgD transcripts studied correspond to the membrane form and, just as in other teleosts, are chimeric, with the inclusion of $\mathrm{C} \mu 1$ and six $\mathrm{C} \delta$ exons. The $\mathrm{C} \mu 1$ exon permits covalent association with light chains, this kind of splicing ( $\mathrm{C} \mu 1$ to $\mathrm{C} \delta$ exon) is not only restricted to teleosts as it has recently been described in porcine IgD transcripts. One interesting feature is that medaka transcripts lack the canonical $C \delta 5$ exon and this finding is confirmed in the genomic sequence, where IGHD loci seem to have been subjected to dramatic recombination events leading to loss of the C $\delta 5$ exon. A high diversity in IGHD genes has been described in teleosts $[39,40]$. Seven $C \delta$ domains comprise the backbone of many bony fish delta chains, wherein a wide range of domain organization within fish lineages is observed. In the Japanese flounder (Paralichthys olivaceus) [41] and stickleback (Gasterosteus aculeatus) [17], the IGHD locus consists of the $\mathrm{C} \delta 1-\mathrm{C} \delta 2-\mathrm{C} \delta 3-\mathrm{C} \delta 4-\mathrm{C} \delta 5-\mathrm{C} \delta 6-\mathrm{C} \delta 7-$ TM1-TM2 exons, in which the homology of domains $\mathrm{CH} 2-\mathrm{CH} 5, \mathrm{CH} 3-\mathrm{CH} 6$ and $\mathrm{CH} 4-\mathrm{CH} 7$ suggests that $\mathrm{C} 82-$

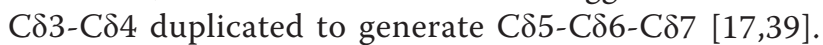
However, in Atlantic salmon (Salmo salar), grass carp (Ctenopharyngodon idella) and catfish (Ictallurus punctatus) a duplication of $\mathrm{C} \delta 2-\mathrm{C} \delta 3-\mathrm{C} \delta 4$ has been described $[15,34,42]$. In Atlantic cod (Gadus morhua) the IGHD locus has undergone rearrangement events leading to the loss of $\mathrm{C} \delta 3, \mathrm{C} \delta 4, \mathrm{C} \delta 5$ and $\mathrm{C} \delta 6$ exons with a tandem duplication of the $\mathrm{C} \delta 1-\mathrm{C} \delta 2$ region. It appears that diversification of IgD may be due to germline changes that are species specific rather than due to different splicing pattern as described in IgM. Therefore, only in sharks partly of IgD, like W heavy chain, is diversified through alternative splicing. Further studies are needed to understand the reason for this phenomenon and the biologi$\mathrm{cal} /$ evolutive meaning of both mechanisms to generate antibody diversity.

Analysis of ESTs showed that there were atypical IgM and IgD transcripts (approx. 15\%), which had stop codons interrupting the reading frames. Most of them lacked the $\mathrm{VH}$ region and contained a genomic sequence, named exon 0 , at the $5^{\prime}$ location, which is spliced directly to the constant exons. It is common to find sterile transcripts from light chain loci in teleosts, and these may be associated to the high frequency of enhancers in the IgL loci of bony fishes $[43,44]$. Recently, unusual IgD transcripts have also been described in Salmo salar [45], wherein the VH and JH sequences are not obvious and include genomic sequences. In catfish [13], in which the $C \delta 1$ is directly 
spliced to leader exon, which was shown to be functional and capable of mediating secretion of IgD from catfish B cells. The authors suggest the possibility that this secreted IgD functions as a pattern-recognition molecule. These results observed in the several teleost species suggest an evolutive and functional role for nontraditional VHDJH rearrangement and needs to be studied in the future. In medaka, the splicing between exon 0 and the rest of the exons indicate that all components of the immunoglobulin heavy chain, except the $\mathrm{VH}$ region, are needed for a specific process in the teleosts.

The ESTs encoding medaka IgM present differences in their $\mathrm{C} \mu$ nucleotide sequences, suggesting a duplicated IGH locus in medaka. Therefore, when we scanned the medaka genome with these ESTs we found a very complex locus, with five tandem duplicated $C \mu$. and $C \delta$ genes separated by $\mathrm{VH}, \mathrm{D}$ and $\mathrm{JH}$ segments. In other fishes we can find duplicated IGH loci, like in I. punctatus, G. aculeatus, S.salar $[17,19,40,45]$ or, like in zebrafish (Danio rerio), only one IGH copy [46]. Duplicated segments in medaka showed a high DNA level homology for exons and introns. The most probable explanation is such duplications occurred recently and take place frequently. In the future, it would be of interest to identify the mechanism responsible for this genetic exchange. Preliminary data indicates the presence of short repeated sequences (SRS)s at the beginning of duplications suggesting their involvement in such exchange processes (data not shown).

The current medaka whole genome sequence draft presents a number of gaps that do not permit exact delineation of gene configuration. Just like in the case of other vertebrates, the IGH locus has regions that are quite difficult to sequence, due to the frequent presence of SRS. Additionally, the analysis of the medaka germline IGH locus gave rise to uncertainties which on the one hand suggested the lack of $\mathrm{C} \mu 3$ and on the other identified $C \delta 7$ as a pseudogene. The database of ESTs and the recently released next generation sequence data from Illumina enabled us to confirm the presence of $\mathrm{C} \mu 3$ and $C \delta 7$ as functional exons. However, the high sequence homology between the duplicated segments prevented us from providing a gap-free IGH locus annotation using this additional information.

Despite the medaka IGH locus having many genes, no genes for IgT/Z have been so far identified as has been the case in catfish [14]. Furthermore, we found exons and even entire zones (Ex. zone 3) that were not expressed. It is difficult to explain the evolutive significance of the presence of exons, which are predicted to be functional (without stop codon or any other alteration in their sequences) but are not going to be expressed. Perhaps the screening and sequencing of EST libraries was not sensitive enough to detect mRNA in low concentrations. However, it seems improbable that zone 3 could generate a functional antibody. A possible explanation for sequence maintenance would be its relationship with the genetic locus structure itself. The high number of recombinations may determine that the predicted functional exons cannot generate antibodies in the medaka strain studied, even though antibodies were expressed by non-homologue recombination in other medaka fish. In order to verify this hypothesis, the sequencing of these loci in other fish strains of the same species should give us different haplotypes.

IGH locus duplications appear to be common in teleost fishes and should be favoured by natural selection. These observations indicate that these duplications may have arisen in a common ancestor teleost or are due to independent gene duplications that occurred in each specie through their specific phylogenetic history. The fact that many teleosts appear to harbor duplications may support the first hypothesis, however there are also data that suggest an independent evolution in different lineages. The high homology between different zones of the IGH locus (as exons as introns) indicates recent duplications processes. However, if they took place a long time ago, then recombinations events would be required to explain sequence maintenance. In medaka, such duplications and recombinations could explain the presence of immunoglobulin constant exons in germline IGH locus, which are apparently functional but are not expressed. The same reasoning can be applied in the case of VH segments, to explain high homology between members of the same family. Thus, all chromosome segments that contain the IGH locus would be subjected to such duplication and recombination processes.

Duplicated genes have been identified in many teleostean fishes and it has been suggested that species diversity might be related to large-scale independent gene duplications or to whole genome duplication in an ancient teleost $[47,48]$. In the case of IGH locus several particular issues remain to be explored. The mechanism known as allelic exclusion prevents the production of more than one specificity in a single lymphoid cell, only one rearrangement product of immunoglobulin is transcribed and translates [49]. Studies of the allelic exclusion of immunoglobulin genes have been performed in species in which a single IGH locus undergoes somatic rearrangement through the lymphocyte development. However, the mechanisms by which teleosts such as medaka, stickleback, catfish, salmo with several IGH locus duplications can exhibit allelic exclusion remains unknown. In medaka, there are at least four IGH duplications that are functional. This means that one cell has the possibility to produce four heavy chains at the same time and therefore could deviate substantially from the clonal selection theory. Eason et al. [50], identified 
different productive gene transcripts in isolated single peripheral blood lymphocytes from cartilaginous fish (Raja eglanteria), indicating the possibility of simultaneous immunoglobulin heavy chains expression from multiple different IGH loci in fishes. In cartilaginous fishes, the IGH locus is arranged in multiple independent clusters, thus indicating that the regulation of immunoglobulin expression could be very different from teleost fishes in which the IGH locus is typically in translocon configuration. The fundamental question regarding the establishment and maintenance of haplotype exclusion in a complex multi-cluster- translocon system such as found in medaka IGH locus remains unanswered today.

Further studies are required to a) understand whether IGH locus duplications involve additional biological mechanisms in the immune system and $b$ ) to gauge the potential evolutive advantages of such configurations to the generation of immunoglobulin diversity in these species.

\section{Conclusions}

The present study shows the genomic organization of the IGH locus in medaka that has genes for IgM and IgD however, no $C \tau$ genes have been identified upstream of the $\mathrm{C} \mu$ region. This IGH locus is very complex, with five duplications that present high homology, being four of them functional. Our results suggest that the IGH locus duplication is very common among teleosts, wherein the existence of a recombination process explains the sequence homology between them.

\section{Additional material}

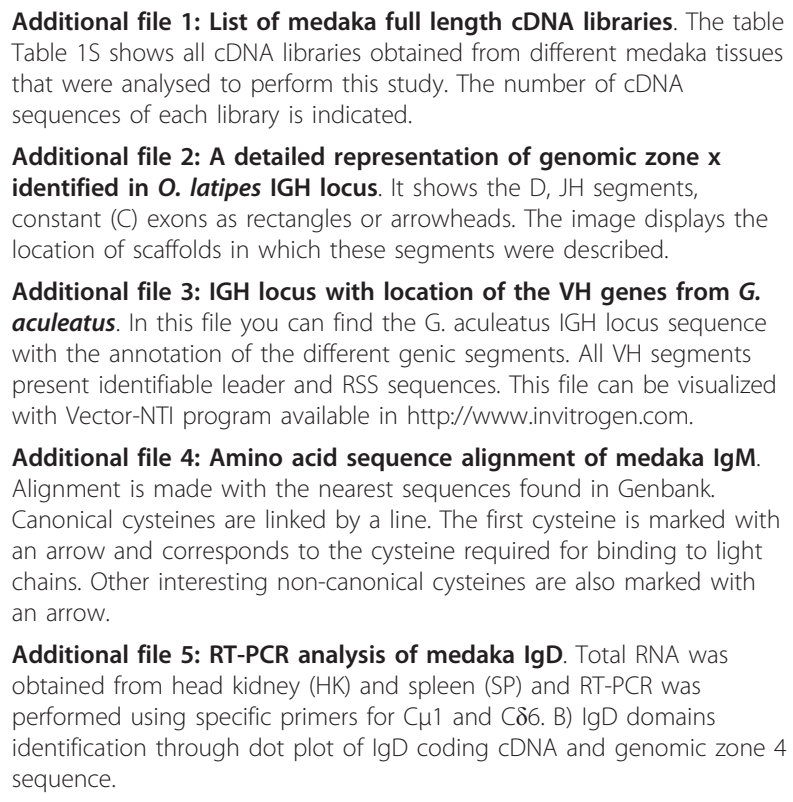

Additional file 5: RT-PCR analysis of medaka IgD. Total RNA was obtained from head kidney (HK) and spleen (SP) and RT-PCR was performed using specific primers for $C \mu 1$ and C $\delta 6$. B) IgD domains identification through dot plot of $\lg D$ coding $C D N A$ and genomic zone 4 sequence.

Additional file 6: Medaka germline nucleotide sequences. In this file you can find nucleotide sequences of different zones described in the medaka IGH locus. The IGHVs file contains the annotation of all VHs. All files can be visualized with Vector-NTI program available in http://www. invitrogen.com.

Additional file 7: Phylogenetic trees. This figure show unrooted phylogenetic trees made with domains of $O$. latipes and $G$. aculeatus $\delta$ left) and $\mu$ (right) chains. MEGA4 software, minimum evolution algorithm and JTT matrix were used to draw the tree. Differences by site are activated with gamma parameter 2.5. ${ }^{*}$ The G. aculeatus $C 85$ does not present any orthologous exon in medaka IGH locus.

Additional file 8: IGH locus diagram with location of the VH genes from 0 . latipes. All genes were localized as indicated in material and methods (top left). Alignment of aminoacid sequences deduced from $\mathrm{VH}$ genes are at the bottom. The alignment was performed and scored according to recommendations of the IMGT. Naming was done using nomenclature proposed by the same organization. Phylogenetic tree of the $\mathrm{VH}$ regions from $\mathrm{O}$. latipes is shown on the top right hand side. MEGA4 software, minimum evolution algorithm and JTT matrix were used to draw the tree. Differences by site are activated with gamma parameter 2.5.

Additional file 9: Confirmation of different genomic zones through genomic short reads alignment. This file shows the result obtained after aligning medaka genomic short reads (DRA000220) with the region of zone 1 that presents $\mathrm{JH}$ segments and the IGHM gene. Alignment details of short reads with the $\mathrm{C} \mu 1$ are indicated. It includes the specific nucleotide sequence to each zone. Identical nucleotides are shown in the same colour (A: green, C: brown, G: lilac, T: blue) and differences are in red and underlined.

\section{Acknowledgements}

The authors would like to thank Drs. J. Cerdá ( Institute of Marine Sciences of Barcelona, CSIC, and Aquaculture Centre) and J. Rotllant (Institute of Marine Sciences of Vigo, CSIC) for providing medaka fishes to perform these studies. We are also very grateful to Anastasia Zimmerman (Grice Marine Laboratory - College of Charleston) for a critical appreciation of the manuscript.

\section{Author details}

'Oceanographic Center of Vigo, Spanish Institute of Oceanography (IEO), Subida a Radio Faro 50, 36390 Vigo, Pontevedra, Spain. ${ }^{2}$ Shared Unit of Immunology, University of Vigo - Vigo University Hospital Complex (Hospital Meixoeiro), Edificio de Ciencias Experimentales, Rua das Abeleiras, Campus As LagoasMarcosende, Vigo 36310, Pontevedra, Spain. ${ }^{3}$ Unidad de Inmunología, Hospital do Meixoeiro, Servizo Galego de Saude (SERGAS), Carretera de Madrid s/n, Vigo 36210, Pontevedra, Spain.

\section{Authors' contributions}

SMM and FGD designed the study, carried out gene annotation, sequences analysis and molecular labwork. CSE participated in sequences alignment and gene annotation. All authors participated in writing the manuscript and read and approved the final version.

Received: 8 March 2011 Accepted: 15 June 2011

Published: 15 June 2011

\section{References}

1. Holland PWH: Gene duplication: Past, present and future. Semin Cell Dev Biol 1999, 10(5):541-547.

2. Shimeld S, Holland P: Vertebrate innovations. Proc Natl Acad Sci USA 2000, 97(9):4449-4452

3. Hsu E, Pulham N, Rumfelt LL, Flajnik MF: The plasticity of immunoglobulin gene systems in evolution. Immunol Rev 2006, 210(1):8-26.

4. Criscitiello MF, Flajnik MF: Four primordial immunoglobulin light chain isotypes, including lambda and kappa, identified in the most primitive living jawed vertebrates. Eur J Immunol 2007, 37(10):2683-2694. 
5. Flajnik MF, Kasahara M: Origin and evolution of the adaptive immune system: genetic events and selective pressures. Nature Reviews Genetics 2010, 11(1):47-59.

6. Harding FA, Amemiya CT, Litman RT, Cohen N, Litman GW: Two distinct immunoglobulin heavy chain isotypes in a primitive, cartilaginous fish, Raja erinacea. Nucleic Acids Res 1990, 18(21):6369-6376.

7. Harding FA, Cohen N, Litman GW: Immunoglobulin heavy chain gene organization and complexity in the skate, Raja erinacea. Nucleic Acids Res 1990, 18(4):1015-1020.

8. Tonegawa S: Somatic generation of antibody diversity. Nature 1983, 302(5909):575-581.

9. Tonegawa S: Proceedings: Determination of the number of antibody structural genes by DNA-RNA hybridization. Hoppe Seylers Z Physiol Chem 1976, 357(5):617.

10. Rumfelt LL, Lohr RL, Dooley H, Flajnik MF: Diversity and repertoire of IgW and IgM VH families in the newborn nurse shark. BMC Immunol 2004, $5(1): 8$.

11. Ota $T$, Rast JP, Litman GW, Amemiya CT: Lineage-restricted retention of a primitive immunoglobulin heavy chain isotype within the Dipnoi reveals an evolutionary paradox. Proc Natl Acad Sci USA 2003, 100(5):2501-2506.

12. Anderson MK, Strong SJ, Litman RT, Luer CA, Amemiya CT, Rast JP, Litman GW: A long form of the skate IgX gene exhibits a striking resemblance to the new shark IgW and IgNARC genes. Immunogenetics 1999, 49(1):56-67.

13. Edholm ES, Bengte'n E, Stafford JL, Sahoo M, Taylor EB, Miller NW Wilson M: Identification of two lgD+ B cell populations in channel catfish, Ictalurus punctatus. J Immunol 2010, 185(7):4082-4094.

14. Bengte'n E, Quiniou S, Hikima J, Waldbieser G, Warr GW, Miller NW, Wilson M: Structure of the catfish IGH locus: analysis of the region including the single functional IGHM gene. Immunogenetics 2006, 58(10):83, 1-844.

15. Hordvik I: Identification of a novel immunoglobulin delta transcript and comparative analysis of the genes encoding lgD in Atlantic salmon and Atlantic halibut. Mol Immunol 2002, 39(1-2):85-91.

16. Danilova N, Bussmann J, Jekosch K, Steiner LA: The immunoglobulin heavy-chain locus in zebrafish: identification and expression of a previously unknown isotype, immunoglobulin Z. Nat Immunol 2005, 6(3):295-302.

17. Gambón-Deza F, Sánchez-Espinel C, Magadán-Mompó S: Presence of an unique IgT on the IGH locus in three-spined stickleback fish (Gasterosteus aculeatus) and the very recent generation of a repertoire of VH genes. Dev Comp Immunol 2010, 34(2):1, 14-122

18. Hansen JD, Landis ED, Phillips RB: Discovery of a unique Ig heavy-chain isotype $(\lg T)$ in rainbow trout: Implications for a distinctive $B$ cell developmental pathway in teleost fish. Proc Natl Acad Sci USA 2005, 102(19):6919-6924

19. Savan $R$, Aman A, Nakao M, Watanuki H, Sakai M: Discovery of a novel immunoglobulin heavy chain gene chimera from common carp (Cyprinus carpio L.). Immunogenetics 2005, 57(6):458-463.

20. Taniguchi Y, Takeda S, Furutani-Seiki M, Kamei Y, Todo T, Sasado T, Deguchi T, Kondoh H, Mudde J, Yamazoe M, Hidaka M, Mitani H, Toyoda A, Sakaki Y, Plasterk RH, Cuppen E: Generation of medaka gene knockout models by target-selected mutagenesis. Genome Biol 2006, 7(12):R1-16.

21. Ozato K, Wakamatsu Y: Developmental Genetics of Medaka. Development Growth and Differentiation 1994, 36(5):437-443.

22. Krumsiek J, Arnold R, Rattei T: Gepard: a rapid and sensitive tool for creating dotplots on genome scale. Bioinformatics 2007, 23(8):1026-1028.

23. Blankenberg D, Von K, Coraor N, Ananda G, Lazarus R, Mangan M, Nekrutenko A, Taylor J: Galaxy: a web-based genome analysis tool for experimentalists. Curr Protoc Mol Biol 2010.

24. Goecks J, Nekrutenko A, Taylor J: Galaxy: a comprehensive approach for supporting accessible, reproducible, and transparent computational research in the life sciences. Genome Biol 2010, 11(8).

25. Milne I, Bayer M, Cardle L, Shaw P, Stephen G, Wright F, Marshall D: Tabletnext generation sequence assembly visualization. Bioinformatics 2010, 26(3):401-402

26. Stanke M, Steinkamp R, Waack S, Morgenstern B: AUGUSTUS: a web server for gene finding in eukaryotes. Nucleic Acids Res 2004, 32(Web Server issue):W309-W3 12.
27. Jung D, Giallourakis C, Mostoslavsky R, Alt FW: Mechanism and control of $\mathrm{V}(\mathrm{D}) \mathrm{J}$ recombination at the immunoglobulin heavy chain locus. Annu Rev Immunol 2006, 24:541-570.

28. Lefranc MP, Pommié C, Kaas Q, Duprat E, Bosc N, Guiraudou D, Jean C, Ruiz M, Da Piédade I, Rouard M, Foulquier E, Thouvenin V, Lefranc G: IMGT unique numbering for immunoglobulin and $T$ cell receptor constant domains and Ig superfamily C-like domains. Dev Comp Immunol 2005 29(3):185-203.

29. Kumar S, Nei M, Dudley J, Tamura K: MEGA: a biologist-centric software for evolutionary analysis of DNA and protein sequences. Brief Bioinform 2008, 9(4):299-306.

30. Ross DA, Wilson MR, Miller NW, Clem LW, Warr GW, Miller NW, Warr GW: Evolutionary variation of immunoglobulin heavy chain RNA processing pathways origins effects and implications. Immunol Rev 1998, 166(1):143-151.

31. Kokubu F, Hinds K, Litman R, Shamblott MJ, Litman GW: Complete structure and organization of immunoglobulin heavy chain constant region genes in a phylogenetically primitive vertebrate. EMBO J 1988, 7(7):1979-1988.

32. Zhao Y, Pan-Hammarström Q, Yu S, Wertz N, Zhang X, Li N, Butler JE, Hammarström L: Identification of IgF, a hinge-region-containing Ig class, and IgD in Xenopus tropicalis. Proc Natl Acad Sci USA 2006, 103(32):12087-12092.

33. Ohta $Y$, Flajnik $M$ : IgD, like $\lg M$, is a primordial immunoglobulin class perpetuated in most jawed vertebrates. Proc Natl Acad Sci USA 2006, 103(28):10723-10728

34. Clem L, Miller N, Warr G, Wilson M, Bengte'n E: Channel catfish immunoglobulins: repertoire and expression. Dev Comp Immunol 2006, 30:77-92.

35. Danilova N, Amemiya CT: Going adaptive: the saga of antibodies. Ann N Y Acad Sci 2009, 1168:130-155.

36. Wilson MR, Ross DA, Miller NW, Clem LW, Middleton DL, Warrt GW: Alternate pre-mRNA processing pathways in the production of membrane IgM heavy chains in holostean fish. Dev Comp Immunol 1995, 19(2):165-177.

37. Lundqvist M, Strömberg S, Bouchenot C, Pilström L, Boudinot P: Diverse splicing pathways of the membrane IgHM pre-mRNA in a Chondrostean, the Siberian sturgeon. Dev Comp Immunol 2009, 33(4):507-515.

38. Coscia MR, Varriale S, Santi CD, Giacomelli S, Oreste U: Evolution of the Antarctic teleost immunoglobulin heavy chain gene. Mol Phylogenet Evol 2010, 55(1):226-233.

39. Hordvik I, Thevarajan J, Samdal I, Bastani N, Krossøy B: Molecular cloning and phylogenetic analysis of the Atlantic salmon immunoglobulin D gene. Scand J Immunol 1999, 50(2):202-210.

40. Savan R, Aman A, Sato K, Yamaguchi R, Sakai M: Discovery of a new class of immunoglobulin heavy chain from fugu. Eur J Immunol 2005, 35(11):3320-3331.

41. Srisapoome P, Ohira T, Hirono I, Aoki T: Genes of the constant regions of functional immunoglobulin heavy chain of Japanese flounder,

Paralichthys olivaceus. Immunogenetics 56(4):292-300.

42. Xiao F, Wang Y, Yan W, Chang M, Yao W, Xu Q, Wang X, Gao Q, Nie P: Ig heavy chain genes and their locus in grass carp Ctenopharyngodon idella. Fish Shellfish Immunol 2010, 29(4):594-599.

43. Edholm ES, Wilson M, Bengte'n E: Immunoglobulin light ( $\lg \mathrm{L})$ chains in ectothermic vertebrates. Dev Comp Immunol 2011, in press.

44. Bao Y, Wang T, Guo Y, Zhao Z, Li N, Zhao Y: The immunoglobulin gene loci in the teleost Gasterosteus aculeatus. Fish Shellfish Immunol 2010, 28(1):40-48.

45. Yasuike M, Boer JD, Schalburg KRV, Cooper GA, McKinnel L, Messmer A So S, Davidson WS, Koop BF: Evolution of duplicated IgH loci in Atlantic salmon, Salmo salar. BMC Genomics 2010, 11:486.

46. Danilova N: Analysis of recombination signal sequences in zebrafish. $\mathrm{Mol}$ Immunol 2005, 42(10):1243-1249.

47. Christoffels A, Koh EGL, Chia JM, Brenner S, Aparicio S, Venkatesh B: Fugu genome analysis provides evidence for a whole-genome duplication early during the evolution of ray-finned fishes. Mol Biol Evol 2004 21(6):1146-1151

48. Brunet FG: Gene loss and evolutionary rates following whole-genome duplication in teleost fishes. Mol Biol Evol 2006, 23(9):1808-1816. 
49. Bergman $\mathrm{Y}, \mathrm{Cedar} \mathrm{H}$ : A stepwise epigenetic process controls immunoglobulin allelic exclusion. Nature Reviews Immunology 2004, 4(10):753-761.

50. Eason DD, Litman RT, Luer CA, Kerr W, Litman GW: Expression of individual immunoglobulin genes occurs in an unusual system consisting of multiple independent loci. Eur J Immunol 2004, 34(9):255, 1-2558.

doi:10.1186/1471-2148-11-165

Cite this article as: Magadán-Mompó et al:: Immunoglobulin heavy chains in medaka (Oryzias latipes). BMC Evolutionary Biology 2011 11:165.

Submit your next manuscript to BioMed Central and take full advantage of:

- Convenient online submission

- Thorough peer review

- No space constraints or color figure charges

- Immediate publication on acceptance

- Inclusion in PubMed, CAS, Scopus and Google Scholar

- Research which is freely available for redistribution

Submit your manuscript at www.biomedcentral.com/submit
() Biomed Central 